\title{
Effect of Core Training on Male Handball Players' Throwing Velocity
}

\author{
by \\ Carmen Manchado ${ }^{1}$, José García-Ruiz', Juan Manuel Cortell-Tormo ${ }^{1}$, \\ Juan Tortosa-Martínez ${ }^{1}$
}

In handball, throwing velocity is considered to be one of the essential factors in achieving the ultimate aim of scoring a goal. The objective of the present study was to analyze the effect of a core training program on throwing velocity in 30 handball players (age $18.7 \pm 3.4$ years, body height $179.3 \pm 7.0 \mathrm{~cm}$, body mass $78.9 \pm 7.7 \mathrm{~kg}$ ), 16 of whom were in the junior category and 14 of whom were in the senior category. The 30 players were randomly divided into two groups, the control group $(n=15)$ and the experimental group $(n=15)$. For a period of ten weeks, both groups attended their regular handball training sessions (four per week), but in addition, the experimental group participated in a program specifically aimed at progressively strengthening the lumbo-pelvic region and consisting of seven exercises performed after the general warm-up in each regular session. Pre- and post-tests were carried out to analyze each player's throwing velocity from different throwing positions and thus assess the effects of this specific training program. Statistically significant differences $(p \leq 0.05)$ in throwing velocity were observed between the experimental group, which presented a percentage improvement of $4.5 \%$, and the control group, which did not show any improvement. The results seem to indicate that an increase in the strength and stability of the lumbo-pelvic region can contribute to an improvement in the kinetic chain of the specific movement of throwing in handball, thus, increasing throwing velocity.

Key words: Lumbo-pelvic region, team sports, performance factors, kinetic chain.

\section{Introduction}

In handball, as in other team sports, shooting a ball at the goal is the culmination of an offensive phase. Success or failure depends on whether a team attains its ultimate aim, that of scoring a goal. Throwing efficiency is the key to winning or losing matches and has been the subject of various studies (García et al., 2011; Marques et al., 2007). Throwing efficiency depends largely on the accuracy and speed of a throw (Gorostiaga et al., 2004). According to various studies (Joris et al., 1985; Manchado et al., 2013; Van Muijen et al., 1991), the factors that determine throwing velocity are technique, coordination and maximum explosive power of the muscles in the upper and lower body, hence the importance of developing training methods that improve both accuracy and throwing velocity.

Throws in which the aim is to reach a high velocity in the most distal segment use a pattern of movement based on kinetic chains (proximal-distal) (Gutiérrez-Dávila et al., 2012). According to studies by Herring and Chapman (1992) and Putnam (1993), the efficiency of the kinetic chain process depends on three broad factors: 1 . the position of the segments in space, 2. participation of the muscles involved, and 3. sequence of participation of the various segments,

1 - Faculty of Education. University of Alicante, Spain. 
which in turn is affected by the transfer of angular impulses from the most proximal segment to the most distal one.

One of the factors that influence the speed of the kinetic chain when throwing is the participation of the musculature involved, including the core muscles. The core musculature includes the trunk and pelvic muscles, which are responsible for maintaining spinal and pelvic stability and help generate and transfer energy from large to small body parts. Core stability is the ability to control the position and motion of the trunk over the pelvis and legs to allow optimum production, transfer and control of force and motion to the terminal segment in integrated kinetic chain activities (Kibler et al., 2006).

Research has established that core training has a positive effect on rehabilitation from injury and reduction of back pain (Danneels et al., 2001; Koumantakis et al., 2005; McGill, 2003). Several studies have been conducted on the effect of core training on performance factors in various sports, but their results are inconsistent. Pedersen et al. (2006) found that after 8 weeks of core muscle training, elite soccer players increased their kicking velocity. Seiler et al. (2006) reported improved club head velocity in junior golf players after a 9 week period of training. In contrast, Schibek (1999) and Stanton et al. (2004), who studied swimming and competitive running, respectively, found no improvement in performance after a period of lumbo-pelvic region training, but did observe a significant improvement in the stability of this region. In handball, the only study that we found in the literature was that conducted by Saeterbakken et al. (2011) on junior female players. Their study showed that after 6 weeks of specific core muscle training, the velocity of the handball players' throws at the goal improved, probably due to the influence of the program on the kinetics of the throwing movement. To the authors' knowledge, there are no studies including male handball players or senior players in the sample.

Given these inconsistencies in the literature regarding the effect of core training on sports performance, together with the paucity of scientific studies in the field of team sports such as handball, the aim of the present study was to determine whether a core training program consisting of exercises aimed at improving the kinetics of movement could lead to an improvement in handball players' throwing velocity.

\section{Material and Methods}

\section{Participants}

The study sample consisted of 30 male handball players (age 18.7 \pm 3.4 years, body height $179.3 \pm 7.0 \mathrm{~cm}$, body mass $78.9 \pm 7.7 \mathrm{~kg}$ ) who were randomly divided into two groups, an experimental group, $\mathrm{E}(\mathrm{n}=15$, age $18.5 \pm 3.0$ years, body height $179.2 \pm 6.5 \mathrm{~cm}$, body mass $78.1 \pm 5.9$ $\mathrm{kg})$, and a control group, $\mathrm{C}(\mathrm{n}=15$, age $18.9 \pm 3.8$ years, body height $179.3 \pm 7.6 \mathrm{~cm}$, body mass 79.8 $\pm 9.2 \mathrm{~kg}$ ).

Prior to commencing the study, players were informed about the training process, the tests that would be carried out as well as the purpose of the study, and gave their signed informed consent to participation in the study, which was signed by a parent or a legal guardian in case of those who had not reached the age of maturity (18 years). Approval for the study was obtained from the Ethics Committee at the University of Alicante, in accordance with the 1975 Declaration of Helsinki. Players had to meet a series of inclusion criteria: be injury-free, have competed on the team throughout that season and be available for competitive handball throughout the period of the experiment. In addition, the selected subjects had to commit to completing the training process in full, following the researcher's guidelines at all times.

For a period of ten weeks, both groups attended their regular handball training sessions (four per week), but in addition, group E participated in a specific and progressive core training program, performed after the general warm-up during each training session.

\section{Measures and procedures}

Throwing test protocol

A radar (StalkePro Inc., Plano), with recording frequency of $33 \mathrm{~Hz}$ and sensitivity of $0.045 \mathrm{~m} \cdot \mathrm{s}^{1}$, was used to measure upper body throwing velocity. The reliability of the test had been studied previously (Duaty et al., 2005; Marques and González-Badillo, 2006). Specific warm-up exercises were performed 15 min prior to the test and they consisted of $5 \mathrm{~min}$ vegetative activation, active stretching and sub-maximal throws. After the warm-up, players were asked to 
throw a ball at maximum velocity without feinting. The test consisted of throwing from four different positions: (1) from the penalty position (7 m); (2) a standing throw without a run-up from the free-throw line $(9 \mathrm{~m})$; (3) a standing throw with a run-up from $9 \mathrm{~m}$; and (4) a jump throw with a run-up from $9 \mathrm{~m}$. Each throw was performed with and without the intervention of a goalkeeper. Each series of throws comprised three attempts, with a $3 \mathrm{~min}$ rest interval between each set. In each case, the best of the three attempts was recorded for further analysis. All throws were performed with an official IHF ball and the use of resin was permitted. After each throw, players were informed of the velocity attained to increase their motivation (García et al., 2011).

Load quantification method

The specific program for core stability carried out by group E included a progressive load (volume, intensity and density), which was quantified using the objective load equivalents method (ECOs according to its Spanish initials) (Cejuela and Esteve-Lanao, 2011). This quantification method was selected since it provides an option for calculating the load in pelvic girdle and strength exercises, unlike others such as the training impulse model (TRIMPS) (Banister, 1991), which do not have a specific method for this type of quantification.

Training process

Group E participated in a core training program for a period of 10 weeks; it consisted of three sessions performed after the general warmup during three of the team's regular training sessions. Each of the specific core sessions consisted of seven exercises focused on improving the kinetic chain of movement involved in the action of throwing, one for the lower part of the rectus abdominis, two for the middle region (external and internal oblique muscles), two for the upper part of the rectus abdominis and two for the posterior region (lumbar and gluteal muscles). According to Has et al. (2001), muscular strength and endurance can be developed through both dynamic and static exercises, including trunk flexion exercises (Picture 1), lateral rotation and flexion (Picture 2) and spinal stabilization exercises (Picture 3). Consequently, each session consisted of an equal amount of both types of exercise. In addition, according to research carried out by Fleck and Schutt (1983), repetition of solely isometric exercises can reduce participant's motivation.

The training process was divided into three stages in order to allow the players to adapt gradually to the program. The initial stage comprised weeks one to three, a period in which low difficulty exercises requiring a low level of technical execution were performed. The sessions lasted for 10-15 minutes, with a total progressive load ranging between 154 and 170 ECOs. The second stage comprised weeks four to seven and consisted of exercises presenting a medium level of technical difficulty. This stage included stability exercises using a Swiss ball. The duration of sessions was 15 to 20 minutes, with a progressive load that ranged between 176 and 228 ECOs. The third and final stage, from weeks eight to ten, consisted of exercises requiring a medium-high level of technical execution and, as in the second stage of training, included stability exercises. These sessions lasted between 20 and 25 minutes, with a total load ranging between 228 and 238 ECOs.

\section{Statistical analysis}

Statistical analysis was performed using the SPSS statistical package version (SPSS 21.0. for Windows). Data distribution was checked by the Shapiro-Wilk test and homogeneity was checked by the Levene test. Baseline group differences analysis between the experimental and control groups was conducted using an unpaired $t$ test for normally distributed variables and the MannWhitney U-test for non-normally distributed variables. In order to assess the main effects of the CORE training program on the throwing velocity, a repeated measures procedure was used in a two-factor analysis of variance (ANOVA) for a composite score of the mean velocity of all throwing types together, and for each throwing type separately. Two time points (effect over time) were considered as the within-participants factor and the differences between the intervention group (CORE training) and the control group (regular training) were treated as a betweenparticipants factor. The box's $\mathrm{M}$ test was applied in order to test the homogeneity of variancecovariance matrices. The effect size was calculated using partial eta ${ }^{2}$.

\section{Results}

The data and general characteristics of 
players in groups $\mathrm{E}$ and $\mathrm{C}$ are shown in Table 1.

Table 2 shows the descriptive statistics for the different throwing position variables in the pre- and post-tests, and the percentage of improvement in each group. Group E, which had participated in additional core training, showed a significant improvement in all throwing tests compared with group C, the control group. Thus, players in the experimental group improved their average throwing velocity compared to the control group when all throwing types were considered together $(p<0.001, \mathrm{ES}=0.644, \mathrm{~F}=$ 49.755). The average percentage of improvement in the post-test compared to the pre-test corresponding to the sum of different throwing variables was $4.3 \%$. When each specific throwing type was analyzed separately, the experimental group improved compared to the control group when performing a standing throw without a runup from the $7 \mathrm{~m}$ line without a goalkeeper $(p<$ $0.001, \quad \mathrm{ES}=0.371, \mathrm{~F}=15.901)$ and with $\mathrm{a}$ goalkeeper $(p<0.001, \mathrm{ES}=0.473, \mathrm{~F}=24.241)$; a standing throw without a run-up from the $9 \mathrm{~m}$ line without a goalkeeper $(p<0.005, \mathrm{ES}=0.292, \mathrm{~F}=$ 11.133 ) and with a goalkeeper $(p<0.05, \mathrm{ES}=0.213$, $\mathrm{F}=7.306$ ); a standing throw with a run-up from 9 $\mathrm{m}$ without a goalkeeper $(p<0.001, \mathrm{ES}=0.387, \mathrm{~F}=$ $17.015)$ and with a goalkeeper $(p<0.001, \mathrm{ES}=$ $0.320, \mathrm{~F}=12.688$ ); and a jump throw with a run-up from $9 \mathrm{~m}$ without a goalkeeper $(p<0.001$, ES $=$ $0.324, \mathrm{~F}=12.941)$ and with a goalkeeper $(p<0.005$, $\mathrm{ES}=0.301, \mathrm{~F}=11.601)$. No significant differences were observed in the control group.

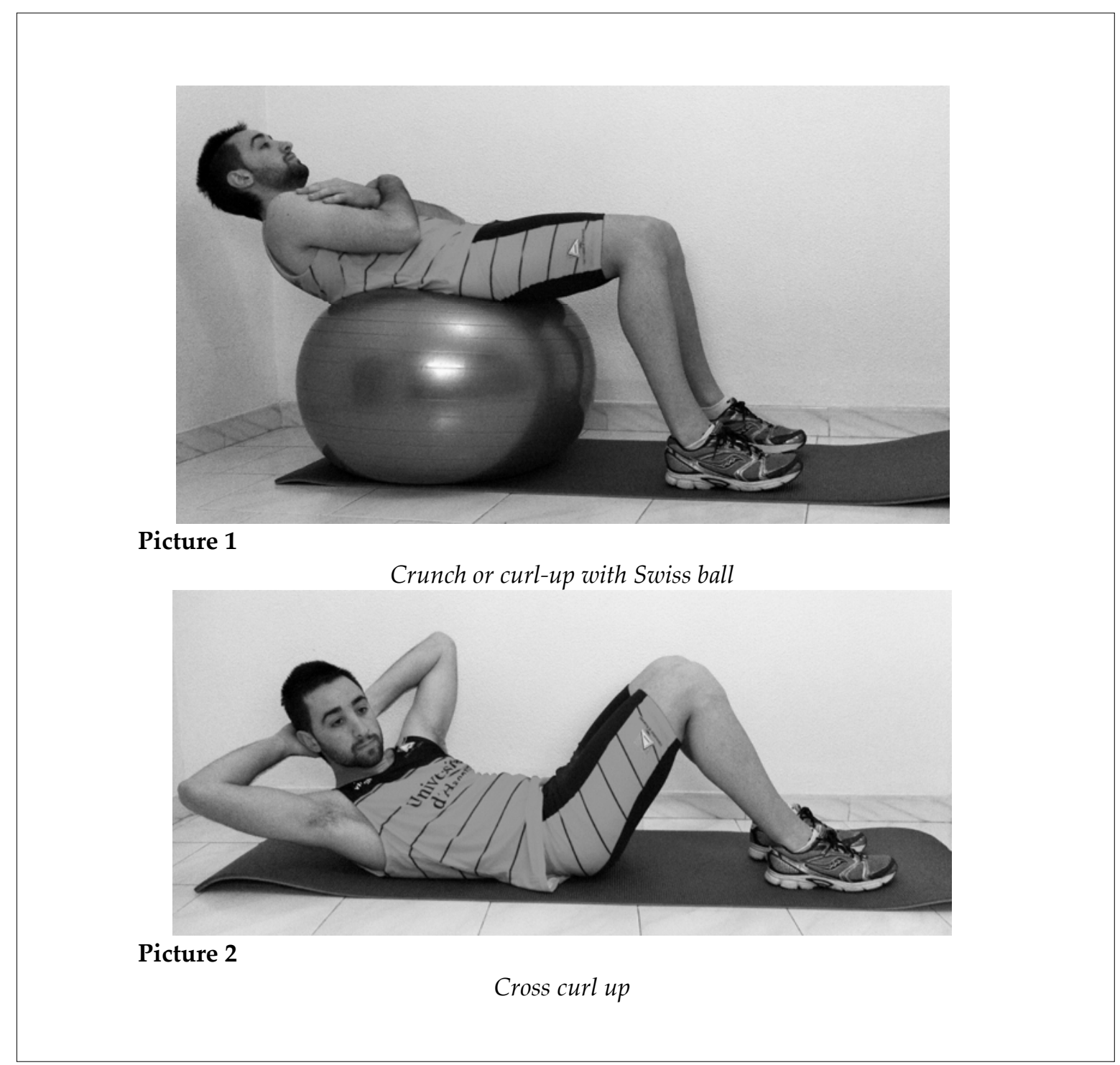




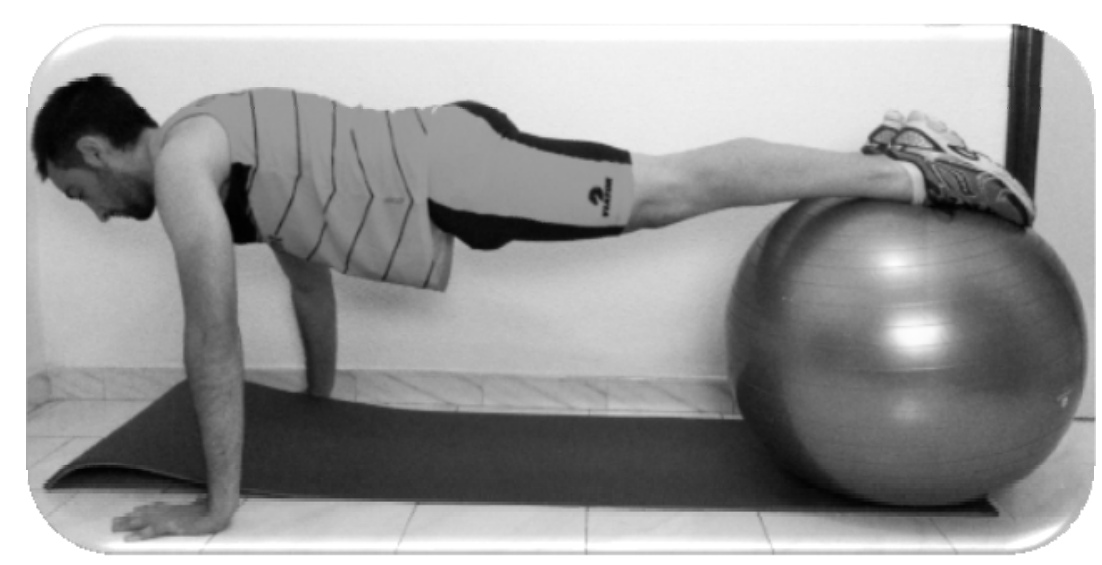

Picture 3

Frontal bridge with Swiss ball

Table 1

Characteristics of players in the Experimental and Control group

\begin{tabular}{llccc}
\hline Participants & $n$ & Age (years) & Body mass $(\mathrm{kg})$ & Height $(\mathrm{cm})$ \\
\hline Experimental & 15 & $18.5 \pm 3.0$ & $78.1 \pm 5.9$ & $179.2 \pm 6.5$ \\
Control & 15 & $18.9 \pm 3.8$ & $79.8 \pm 9.2$ & $179.3 \pm 7.6$ \\
total & 30 & $18.7 \pm 3.8$ & $78.9 \pm 7.7$ & $179.3 \pm 7.0$ \\
\hline
\end{tabular}

Data are mean $\pm S D$

Table 2

Changes in throwing speed of players in Experimental (E) and Control group (C)

\begin{tabular}{lcccccc}
\hline & $\begin{array}{c}\text { Pre-test } E \\
(\mathrm{~km} / \mathrm{h})\end{array}$ & $\begin{array}{c}\text { Post-test } E \\
(\mathrm{~km} / \mathrm{h})\end{array}$ & $\begin{array}{c}\% \\
\text { Variation }\end{array}$ & $\begin{array}{c}\text { Pre-test C } \\
(\mathrm{km} / \mathrm{h})\end{array}$ & $\begin{array}{c}\text { Post-test C } \\
(\mathrm{km} / \mathrm{h})\end{array}$ & $\begin{array}{c}\% \\
\text { Variation }\end{array}$ \\
\hline $7 \mathrm{~m}$. & $76.1 \pm 10.9$ & $80.0 \pm 10.8+$ & $5.12 \%$ & $73.6 \pm 11.6$ & $73.7 \pm 11.3$ & $0.1 \%$ \\
$7 \mathrm{~m}+\mathrm{GK}$ & $75.5 \pm 10.7$ & $79.4 \pm 9.6+$ & $5.16 \%$ & $73.4 \pm 12.1$ & $73.0 \pm 12.3$ & $-0.5 \%$ \\
$9 m$ & $77.8 \pm 10.2$ & $80.8 \pm 10.3^{*}$ & $3.9 \%$ & $74.6 \pm 12.4$ & $74.9 \pm 11.6$ & $0.4 \%$ \\
$9 m+$ GK & $77.2 \pm 11.1$ & $79.6 \pm 10.1^{*}$ & $3.1 \%$ & $74.4 \pm 11.9$ & $73.7 \pm 12.2$ & $-0.9 \%$ \\
$9 m$ 3 steps & $81.8 \pm 12.2$ & $85.7 \pm 11.7+$ & $4.8 \%$ & $79.1 \pm 13.4$ & $79.3 \pm 12.8$ & $0.3 \%$ \\
$9 m$ 3 steps + GK & $83.4 \pm 11.9$ & $86.5 \pm 11.2+$ & $3.7 \%$ & $78.5 \pm 13.0$ & $77.5 \pm 13.5$ & $-1.3 \%$ \\
9 m jump & $80.4 \pm 9.1$ & $83.6 \pm 8.6+$ & $4.0 \%$ & $76.7 \pm 10.9$ & $75.3 \pm 11.5$ & $-1.8 \%$ \\
9 m jump + GK & $79.9 \pm 8.6$ & $83.8 \pm 8.8^{*}$ & $4.9 \%$ & $75.4 \pm 12.2$ & $75.7 \pm 10.9$ & $0.4 \%$ \\
$\quad$ total & & & $4.3 \%$ & & & $-0.4 \%$ \\
\hline
\end{tabular}

Data are mean $\pm S D$

Significant differences at ${ }^{*} p \leq 0.05 .+p \leq 0.001$

$G K=$ Goalkeeper 


\section{Discussion}

The main purpose of this study was to explore the effect of a training program aimed at strengthening the muscles in the lumbo-pelvic region by means of exercises focused on improving the kinetics of movement in handball players' throwing velocity. The results obtained support the initial hypothesis, indicating that a program of this nature leads to an improvement in male players' throwing velocity. Group E showed statistically significant improvements in all throwing positions analyzed, whereas group C presented no statistically significant changes in any of the variables analyzed (Table 2 ).

Based on the hypothesis that an increase in the level of core muscle strength results in an improvement in the kinetic chain of movement, several authors have attempted to apply this to different disciplines. Such is the case of the studies conducted by Pedersen et al. (2006), who showed that after an 8 week period of core training, elite soccer players presented increased kicking velocity. Another study carried out by Seiler et al. (2006) demonstrated improved club head velocity in junior golf players after a 9 week period of training. Van Pletzen and Venter (2012) showed that there was a correlation between the results of the Bunkie test (which measures fascia restrictions in five kinetic chains) and tests which measured rugby players' physical condition: vertical jump performance $(p<0.05 ; \mathrm{r}=0.24)$, repeated sprint ability $(p<0.05 ; \mathrm{r}=0.22)$, Illinois agility $(p<0.05 ; \mathrm{r}=-0.23), 10 \mathrm{~m}$ sprint performance $(p<0.05 ; \mathrm{r}=0.24), 40 \mathrm{~m}$ sprint performance $(p<0.05 ; \mathrm{r}=-0.25)$ and Max pull-ups $(p<0.05 ; \mathrm{r}=0.27)$. Players who showed higher levels of strength in the core muscles and therefore obtained better results in the Bunkie test also performed better in the various physical tests.

The evidence suggests that the increase in group E's throwing velocity was due to an increase in strength of muscles in the lumbopelvic region as a result of core training. In turn, this led to an improvement in the kinetic chain of movement due to increased stability and greater transmission of power and energy between the upper and lower extremities in the action of throwing (Kibler et al., 2006). This increase could be explained by an improvement in the kinetic chain of movement, but also perhaps by an improvement in technique due to core training, another performance factor involved in throwing. As throwing technique was not analyzed, further studies should be carried out to determine the factors influencing this improvement. These results are consistent with those reported in other studies (Barata, 1999; Ettema, 2008; Gorostiaga, 1999) in which strength was improved using the traditional approach of strength training programs (3 x 6RM, 8-12 RM and pyramid training) over a period of 9 weeks, and in which improvements ranging between 1.4 and $6.9 \%$ were obtained.

More specifically, the results of the present study confirm the conclusions reached in a study by Prokopy et al. (2008) on softball players, comparing strength training consisting of open or closed kinetic chain exercises for 12 weeks. A 3.4\% improvement in throwing speed was obtained following an upper body training program using closed kinetic chain exercises, compared with a $0.5 \%$ improvement following a program using open kinetic chain exercises. In their study on the effect of a 6 week core training program on throwing velocity in female junior handball players, Saeterbakken et al. (2011) showed that this type of training lead to an improvement in this variable, obtaining a percentage of improvement of $4.9 \%(p=0.01)$, similar to the percentage $(4.3 \%)$ obtained in the present study on male players in senior and junior categories.

Despite the strong theoretical basis which supports the use of this type of training and the studies cited here that support this theory, inconsistencies still remain in the literature concerning the effect of core training on performance or injury prediction in athletes (Danneels et al., 2001; Koumantakis et al., 2005; McGill, 2003). Poor design of the progression of the selected core exercises may explain why some authors have not observed any effect with regard to improving performance in various sports disciplines (Schibek, 1999; Stanton et al., 2004), although such improvement has been observed in pelvic girdle stability. In our study, subjecting the extremities to increased instability and resistance heightened the difficulty of the exercises, providing sufficient stimulus to core muscles during the training period.

The results of the present study and of those mentioned above confirm the importance of 
strength training in handball and other similar sports, as well as specific exercises to strengthen and improve the stability of core muscles, since the evidence suggests this is associated with an improvement in factors that influence game performance, such as throwing velocity which, together with accuracy, determines performance efficiency (Gorostiaga et al., 2004). Positive results were observed in junior and amateur senior category players alike, although differences might be found if the subjects under study were topclass professional players. Therefore, a future line of research could be to implement a core training program for elite players at the highest standard. Another line could be to compare strength training using weights with specific core training. Meanwhile, training using isometric exercises or a low dynamic component that is carried out in a single plane of motion and employing symmetrical loads provides a very limited stimulus for proprioception and stability (McGill et al., 2003). However, the actions to improve are asymmetrical, involve considerable multiplanar joint movement and require a high degree of skill to maintain balance and dynamic stability (Saeterbakken et al., 2011). Therefore, among other possible future strategies, it would be desirable to include a final training stage focused on movements in dynamic contexts that are strongly related (planes, joints, movement patterns and asymmetrical loads) to the tasks in which an effect is sought.

\section{Conclusions}

A progressive program for strengthening and training the lumbo-pelvic region in order to improve stability and kinetics of movement seems to be related to an increase in handball players' throwing velocity.

Strength training is vitally important in handball since it leads to an improvement in the specific factors that influence game performance. Therefore, it would seem highly advisable to implement strength training programs in handball training and in team sports in general, due to its influence on performance. In addition, and more specifically, it would be advisable to introduce a core training program, since significant potential benefits (injury prevention, improvement in static and dynamic balance, improvement in throwing velocity) can be obtained with a relatively small investment of time (10-15 $\mathrm{min})$.

\section{References}

Banister EW. Modeling human performance. In H.J. Green, J.D. McDougal, \& H. Wenger (Eds), Physiological testing of elite athletes. Champaign, IL: Human Kinetics, 403-424; 1991

Barata J. Changes in ball velocity in the handball free throw, induced by two different speed-strength training programs. Motricidade Humana, 1992; 8(1): 45-55

Cejuela-Anta R, Esteve-Lanao J. Training load quantification. J Hum Sport exercise, 2011; 6: 218-232

Danneels LA, Vanderstraeten GG, Cambier DC, Witvrouw EE, Bourgois J, Dankaerts W, De Cuyper HJ. Effects of three different training modalities on the cross sectional area of the lumbar multifidus muscle in patients with chronic low back pain. Brit J Sport Med, 2001; 35(3): 186-191

Dauty M, Kitar E, Dubois C, Potiron-Josse M. Relationship between ball velocity and the shoulder rotators isokinetic torque in high-level handball players. Sci Sports, 2005; 20 (5-6): 300-303

Ettema G, Glosen T, van den Tillaar R. Effect of specific resistance training on overarm throwing performance. Int J Sports Phys Perf, 2008; 3(2): 164-175

Fleck SJ, Schutt RC. Types of strength training. Orthop Clin N Am, 1983; 14: 449-458

García M, Alcaraz PE, Ferragut C, Manchado C, Abraldes JA, Rodríguez N, Vila H. Body composition and 
throwing velocity in elite female handball players. Cultura, Ciencia y Deporte, 2011; 17: 129-135

Gorostiaga EM, Izquierdo M, Iturralde P, Ruesta M, Ibáñez J. Effects of heavy resistance training on maximal and explosive force production, endurance and serum hormones in adolescent handball players. Eur J Appl Physiol, 1999; 80(5): 485-493

Gorostiaga EM, Granados C, Ibanez J, Izquierdo M. Differences in physical fitness and throwing velocity among elite and amateur male handball players. Int J Sports Med, 2004; 26(3): 225-232

Gutiérrez-Dávila M, Rojas-Ruiz FJ, Ortega-Becerra M, Párraga-Montilla JA, Campos-Granell J. Functional variability as efficiency factor in handball throwing to the goal. Revista de Ciencias del Deporte ebalonmano, 2012; 8(2): 121-134

Has CJ, Feigenbaum MS, Franklin BA. Prescription of resistance training for healthy populations. Sports Med, 2001; 31: 953-964

Herring RM, Chapman AE. Effects of changes in segmental values and timing of both torque and torque reversal in simulated throws. J Biomech, 1992; 25(10): 1173-1184

Joris H, Edwards VM, Van Ingen Schenau GJ, Kemper HCG. Force, velocity and energy flow during the overarm throw in female handball players. J Biomech, 1985; 18(6): 409-414

Kibler WB, Press J, Sciascia A. The role of core stability in athletic function. Sports Med, 2006; 36: 189-198

Koumantakis GA, Watson PJ, Oldham JA. Trunk muscle stabilization training plus general exercise versus general exercise only: Randomized controlled trial of patients with recurrent low back pain. Phys Ther, 2005, 85(3): 209-225

Manchado C, Tortosa J, Vila H, Platen P. Performance factors in female team handball. Physical and physiological aspects - a review. J Strength Cond Res, 2013; 27(6): 1708-1719

Marques MC, González-Badillo JJ. In-season resistance training and detraining in professional team handball players. J Strength Cond Res, 2006; 20(3): 563-571

Marques MC, Van den Tillaar R, Vescovi JD, González-Badillo JJ. Relationship between throwing velocity, muscle power, and bar velocity during bench press in elite handball players. Int J Sports Phys Perform, 2007; 2(4): 414-422

McGill SM, Grenier S, Kavcic N, Cholewicki J. Coordination of muscle activity to assure stability of the lumbar spine. J. Electromyogr. Kinesiol, 2003; 13(4): 353-359

Pedersen JLS, Magnussen R, Kuffel E, Seiler, S. Sling exercise training improves balance, kicking velocity and torso stabilization strength in elite soccer players. Med Sci Sports Exerc, 2006; 38: 243

Prokopy MP, Ingersoll CD, Nordenschild E, Katch FI, Gaesser GA, Weltman A. Closed-kinetic chain upperbody training improves throwing performance of NCAA Division I softball players. J Strength Cond Res, 2008; 22 (6): 1790-1798

Putnam CA. Sequential motions of body segments in striking and throwing skills: Descriptions and explanations. J Biomech, 1993; 26, Supplement 1(0): 125-135

Saeterbakken A, Van Den Tilllar R, Seiler S. Effect of core stability training on throwing velocity in female handball players. J Strength Cond Res, 2011; 25(3): 712-718

Schibek JS, Guskiewicz KM, Prentice WE, Mays S, Davis JM. The effect of core stabilization training on functional performance in swimming. J Athl Train, 1999; 34: 27

Seiler S, Skaanes PT, Kirkesola G. Effects of sling exercise training on maximal clubhead velocity in junior golfers. Med Sci Sports Exerc, 2006; 38: 286

Stanton R, Reaburn PR, Humphries B. The effect of short-term Swiss ball training on core stability and running economy. J Strength Cond Res, 2004; 18: 522-528 
Van Muijen AE, Joris H, Kemper HC, Van Ingen Schenau GJ. Throwing practice with different ball weights: Effects on throwing velocity and muscle strength in female handball players. Sports Training, Medicine $\mathcal{E}$ Rehabilitation, 1991; 2(2), 103-113

Van Pletzen D, Venter R. The relationship between the Bunkie-test and physical performance in Rugby Union Players. Int J Sports Science E Coaching, 2012; 7 (2): 545-555

\section{Corresponding author:}

\section{Carmen Manchado}

University of Alicante

Carretera de San Vicente del Raspeig s/n

03690 San Vicente del Raspeig, Spain

Phone: +34 630730844

Fax: 965903464

E-mail: Carmen.manchado@ua.es 